\title{
COMPLEX DYNAMICS IN LINEAR PI CONTROL SYSTEMS WITH SATURATIONS
}

\author{
DINÁMICA COMPLEJA EN SISTEMAS DE CONTROL LINEALES PI \\ CON SATURACIONES
}

\author{
Guillermo León Gallo Hernández¹, Luis Fernando Castaño Londoño', \\ Gustavo Adolfo Osorio Londoño ${ }^{1}$.
}

${ }^{1}$ Universidad Nacional de Colombia, Sede Manizales. ggalloh@unal.edu.co, lfcastanol@unal.edu.co, gaosoriol@unal.edu.co

\section{RESUMEN}

En este artículo se muestra como las restricciones debidas a saturaciones conducen a respuestas inesperadas en sistemas lineales. Se emplea un modelo basado en máquinas de estado finito para la simulación de un sistema de primer orden con un controlador proporcional-integral (PI) con saturaciones en la acción de control integral y en el actuador. Las simulaciones permiten observar como dichas saturaciones pueden determinar la existencia de seis diferentes configuraciones para el sistema. Resultados numéricos permiten confirmar que la contribución de cada configuración conduce la dinámica del sistema a una órbita periódica aislada. Este comportamiento es validado con resultados experimentales de un servocontrolador PI para un motor DC con anti-windup en la acción de control integral.

Palabras clave: Dinámica del sistema, Controlador PI, Saturaciones, Máquina de estado finito.

\begin{abstract}
In this paper is shown how restrictions due to saturations can lead to unexpected responses in linear systems. A model for systems with saturations based on finite state machines is used to simulate a first order linear system with a proportional-integral (PI) controller with a saturation on the integral branch and on the actuator. The system simulations show how such saturations can determine the existence of six different configurations for the system. Numerical results let confirm that the contributions of each configuration to system dynamics lead to an isolated periodic orbit. This behavior is validated with experimental results of a PI servocontroller for a DC motor with anti-windup saturation on the integral branch.
\end{abstract}

Key words: System dynamics, PI controller, Saturations, Finite state machine. 


\section{INTRODUCTION}

PI control is a classical linear technique used in a wide variety of systems. When this technique is applied to a system, the controller is designed such that a response with the inherent complexity of linear systems is expected. However, it is well known that the system responses have sometimes a complexity only seen in nonlinear systems. This behavior can be caused by system restrictions related to its physical properties (1). Moreover, it also has been reported that restrictions due to saturations presented in real control systems can lead to nonlinear behaviors. Studies about the dynamic and control of linear systems with saturation are developed in (2-8). The most common saturation found in control systems is the magnitude limitation in the total control action. This nonsmooth nonlinearity is generally presented because all control actuation devices are subject to amplitude saturation $(1,9)$. Moreover, when an integral branch is presented in a control system with actuator saturation as shown in Fig. 1, a phenomenon known as integrator windup appears $(1,8,10)$. Analysis and simulations of this phenomenon and different ways to avoid it (antiwindup strategies) are reported in (1,8,10-12). These strategies have been proposed to prevent, among others, certain phenomena in the dynamics of saturated systems as overshoots and limit cycles (13).

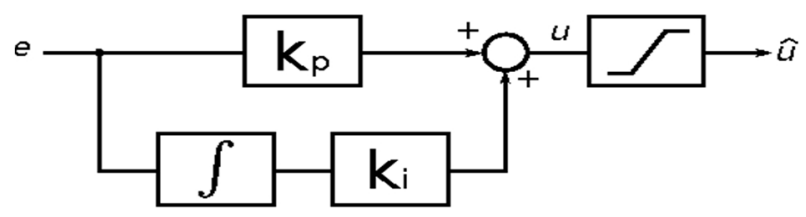

Figure 1: Magnitude limitation in the control action

In this paper, it is shown that when a conditional integration anti-windup method is applied to a saturated system as in Fig. 2, there are nonlinear behaviors under certain conditions. In section 2 , the methodology used to observe the complexity of PI control systems with saturations are presented. First, the numerical methods used for simulations of a first order linear system with a PI controller are detailed, and secondly, the materials and methods used for the experiment are described. In section 3 results and discussion are presented. Through the simulations is shown how saturations in the total control action and the integral branch can generate a coexistence of three possible solutions, one of which is an isolated periodic orbit. Simulation results let observe that each saturation produces abrupt changes in the system, and their interaction generates six possible configurations. It can indeed be shown how the contribution of each configuration to the system dynamic yields to the periodic orbit. Experimental results of a PI servocontroller for a DC motor with actuator saturation and conditional integration anti-windup shown that the same possible solutions are presented in this third order system. Finally, conclusions are given in section 4.

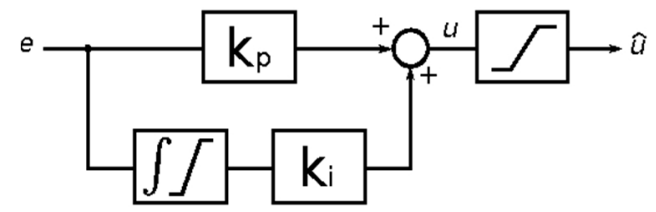

Figure 2: Basic scheme of the conditional integration antiwindup method.

\section{MATERIALS AND METHODS}

\section{NUMERICAL ANALYSIS: FIRST ORDER LINEAR SYSTEM CONTROLLED WITH PI}

Let's consider the system described in equation (1). Here it is shown the dynamic of a linear system of first order controlled with PI.

$$
y=2 y+\hat{u}
$$

Where $\hat{u}=s a t(u)$, and $u$ is the control signal defined as in [2]

$$
u=\left\{\begin{array}{lcc}
k_{p} e-k_{i} h_{\mathrm{int}} & \text { if } & \int e \leq-h_{\mathrm{int}} \\
k_{p} e+k_{i} h_{\mathrm{int}} & \text { if } & -h_{\mathrm{int}}<\int e<h_{\mathrm{int}} \\
k_{p} e+k_{i} h_{\mathrm{int}} & \text { if } & \int e \geq h_{\mathrm{int}}
\end{array}\right.
$$

In which $h_{\text {int }}$ is the maximum value that can take the integrator output. The value of $h_{\text {act }}$ is the maximum level that can take control signal. The constants $k_{p}$ and $k_{i}$ are respectively the proportional and integral constants, and it is the error signal calculated as the difference between the reference input and the system output $y$.

To analyze the response based on the error signal, the system can be represented as shown in (3).

$$
\left[\begin{array}{l}
\dot{x}_{1} \\
\dot{x}_{2}
\end{array}\right]=\left[\begin{array}{cc}
2-k_{p} & -k_{i} \\
1 & 0
\end{array}\right]\left[\begin{array}{l}
x_{1} \\
x_{2}
\end{array}\right]
$$


Thus, due to the saturations presented in the integral branch and the total output of the controller, it is possible to state the existence of six different configurations for the system as shown in (4).

$$
\dot{x}=A_{i} x+B_{i} \quad i=1, \ldots, 6
$$

Therefore, the system can be described with a model based on a finite state machine, where each configuration of the system is associated with a state as shown from (5) to (10).

- State $1\left(\mathrm{~s}_{1}\right)$ : the system is free, it is not saturated

$$
A_{1}=\left[\begin{array}{cc}
2-k_{p} & -k_{i} \\
1 & 0
\end{array}\right] \quad B_{1}=\left[\begin{array}{l}
0 \\
0
\end{array}\right]
$$

- State 2 (s2): only the integrator is saturated

$$
A_{1}=\left[\begin{array}{cc}
2-k_{p} & -k_{i} \\
1 & 0
\end{array}\right] \quad B_{1}=\left[\begin{array}{l}
0 \\
0
\end{array}\right]
$$

- State $3\left(\mathrm{~s}_{3}\right)$ : only the control signal is saturated at its upper level

$$
A_{3}=\left[\begin{array}{ll}
2 & 0 \\
1 & 0
\end{array}\right] \quad B_{3}=\left[\begin{array}{c}
h_{a c t} \\
0
\end{array}\right]
$$

- State $4\left(\mathrm{~s}_{4}\right)$ : only the control signal is saturated in its lower level

$$
A_{4}=\left[\begin{array}{ll}
2 & 0 \\
1 & 0
\end{array}\right] \quad B_{4}=\left[\begin{array}{c}
-h_{a c t} \\
0
\end{array}\right]
$$

- State $5\left(\mathrm{~s}_{5}\right)$ : the integrator and the control signal are saturated at their upper level

$$
A_{5}=\left[\begin{array}{cc}
2 & 0 \\
0 & 0
\end{array}\right] \quad B_{5}=\left[\begin{array}{c}
h_{a c t} \\
0
\end{array}\right]
$$

- State 6 (s6): the integrator and the control signal are saturated at their lower level State 5 (s5): the integrator and the control signal are saturated at their upper level

$$
A_{6}=\left[\begin{array}{ll}
2 & 0 \\
0 & 0
\end{array}\right] \quad B_{6}=\left[\begin{array}{c}
-h_{a c t} \\
0
\end{array}\right]
$$

The state transition diagram of the finite state machine is shown in Fig. 3.

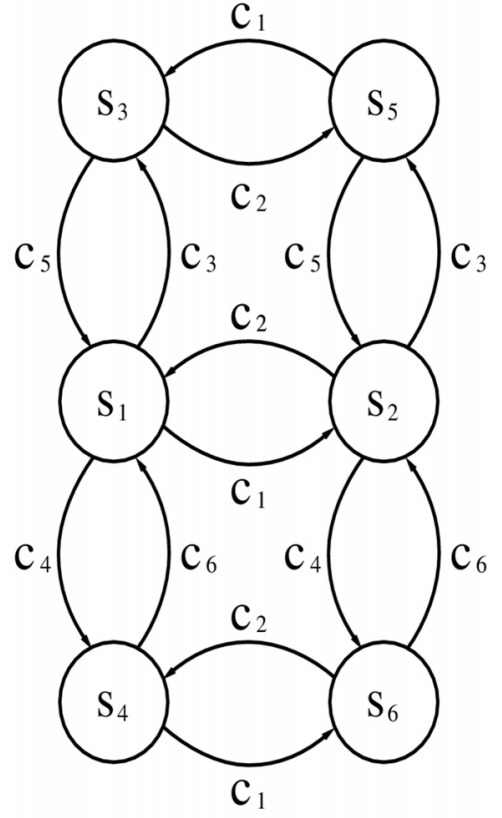

Figure 3: State transition diagram of the finite state machine model.

With these six configurations and establishing the conditions for transition between them, it is possible to simulate the entire system. The conditions for state transitions are defined as follows:

- $\quad c 1$ : $\operatorname{hint}^{2}-x_{2}^{2}=0 \downarrow$, saturate the integrator

- $c_{2}: x_{1}=0 \uparrow \downarrow$, releasing the integrator

- $c_{3}: h_{\text {act }}-\mathrm{u}=0 \downarrow$, saturate the actuator at its upper level

- $c_{4}: h_{a c t}+u=0 \downarrow$, saturate the actuator at its lower level

- $c_{5}: h_{\text {act }}-u=0 \uparrow$, releasing the actuator

- $c_{4}: h_{a c t}+u=0 \uparrow$, releasing the actuator.

The up arrow means that the function is increasing, and the down arrow means that the function is decreasing. If both arrows appear, it is irrelevant whether the function is increasing or decreasing.

\section{EXPERIMENTAL DESIGN: DIGITAL PI SERVO- CONTROLLER FOR A DC MOTOR}

In this work, it has been proposed that some results found in the simulation of the first order system can be extrapolated to the case of a PI servocontroller for a DC motor. The system implemented controls a $24 \mathrm{~V}$ permanent magnet excited brushed DC motor with an incremental encoder for position feedback mounted in the motor shaft. The power stage interface to deliver the 


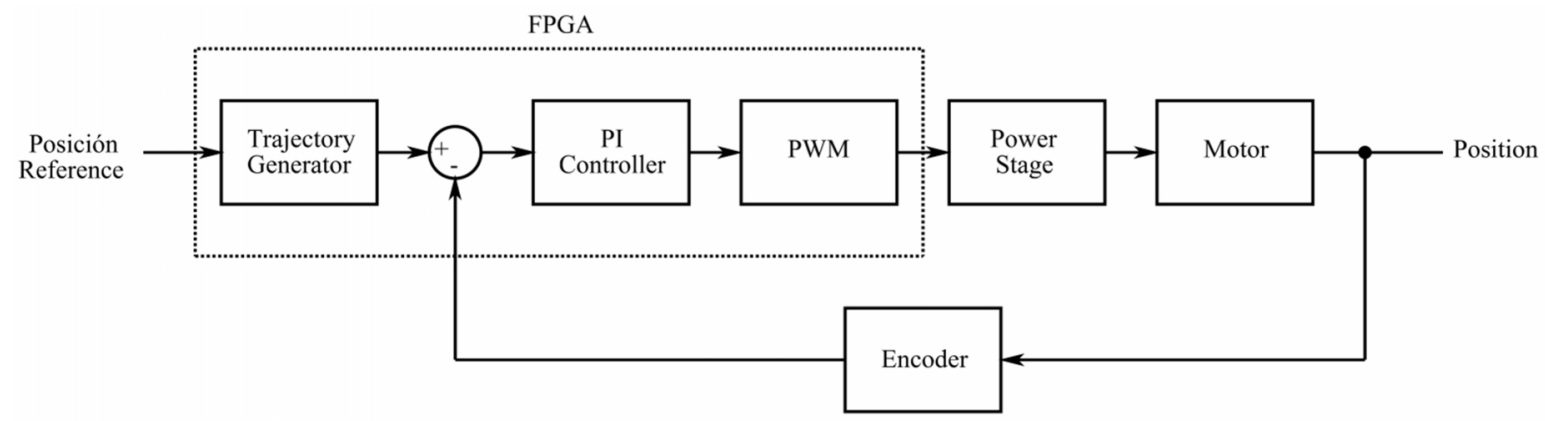

Figure 4: Basic scheme of servo control of a DC motor

current and perform the switching from the PWM signal to the motor is made using a full $\mathrm{H}$-bridge driver in an integrated circuit. Modules for trajectory generation, encoder signal decoding, PI controller and PWM control signal generation are implemented in the FPGA. The block diagram of the servocontroller implemented is shown in Fig. 4.

The control system is implemented in a DE3 Stratix III FPGA board of Terasic Technologies Inc. using Quartus II software of Altera Corporation. Experimental data are obtained using Signal Tap II Logic Analyzer tool available within the Quartus II. With this tool, it has been acquired samples of the motor shaft position, error signal, integral error, and control signal from the FPGA for each experiment.

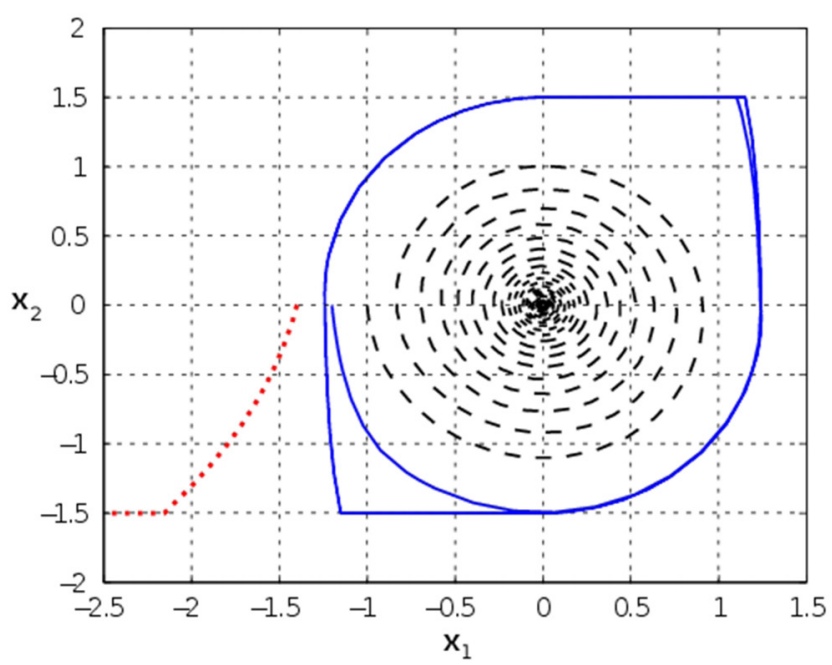

Figure 5: Coexistence of solutions. In the phase portrait, three graphs can be seen. They represent the dynamic for an initial condition for which the system evolves to an equilibrium point located at the origin (dashed line), an initial condition for which the system reaches a periodic orbit (solid line), and an initial condition that makes the system unstable (dotted line).

\section{RESULTS AND DISCUSSION}

\section{NUMERICAL RESULTS}

With the simulations, it can be seen that due to the presence of the two saturations, there is a coexistence of three possible solutions for the system according to the initial conditions as can be observed in the phase portrait shown in Fig 5. For some initial conditions, the system evolves to an equilibrium point located at the origin. For other initial conditions, the system reaches a periodic orbit. In the last case, there are some initial conditions which make the system unstable.

The periodic orbit generated by the interaction of both saturations is shown in Fig. 6. In this phase portrait, the contribution of each configuration in the formation of the periodic orbit can be observed. Also, it can be seen

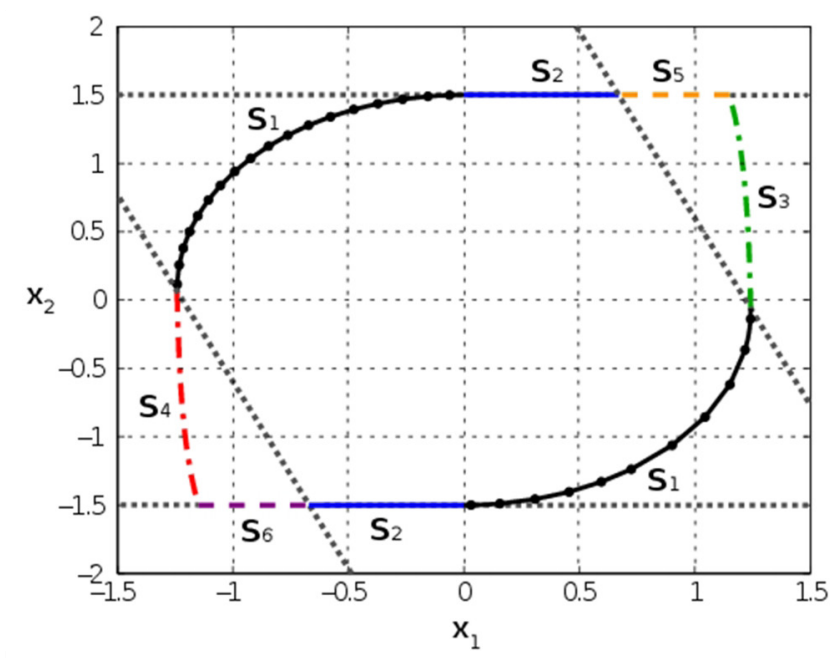

Figure 6: Contribution of each configuration in the formation of the periodic orbit. Horizontal dotted lines represent the integral action saturation. Diagonal dotted lines represent the total control action saturation. Solid lines with dots represent the configurations associated with state s1. Solid lines with state s2. Upper rigth dash-dotted line with state s3. Lower left dash-dotted line with state s4. Upper rigth dashed line with state s5. Lower left dashed line with state $s_{6}$. 
that the integrator saturation (horizontal dotted lines) tends to stabilize the system while the actuator saturation (diagonal dotted lines) makes the system unstable.

\section{EXPERIMENTAL RESULTS}

In the experiments, it has been observed that some initial conditions or disturbances lead the motor shaft to an oscillatory behavior, as shown in Fig. 7. In Fig. 7(a), the motor shaft position is shown. In Fig. 7(b) and Fig. 7(c), the error signal and error integral are shown respectively. In Fig. 7(d) is shown the control signal. In Fig. 7(b) and Fig. 7(c) can be observed that the error integral and control signal are saturated.

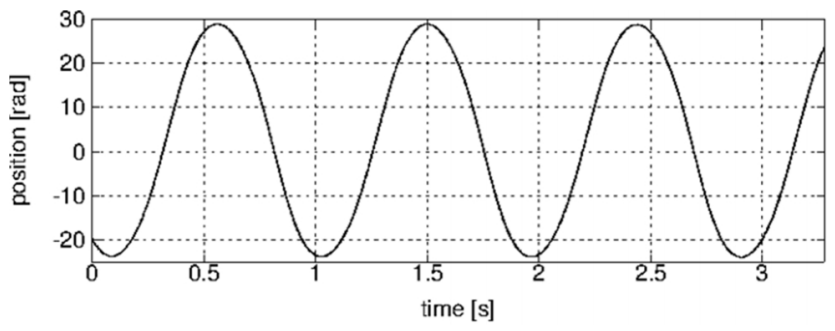

(a) Motor shaft position.

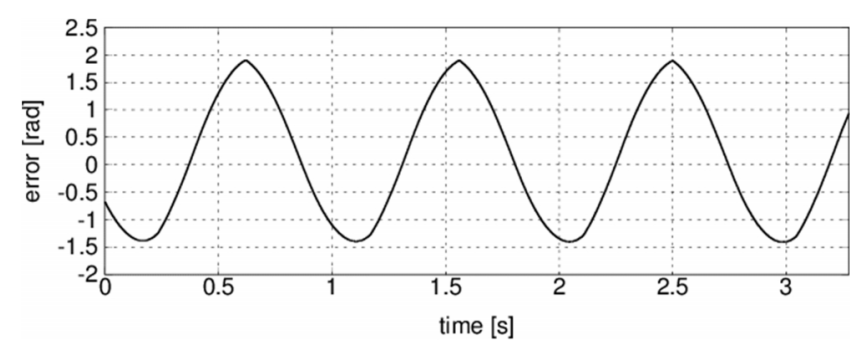

(b) Error signal.

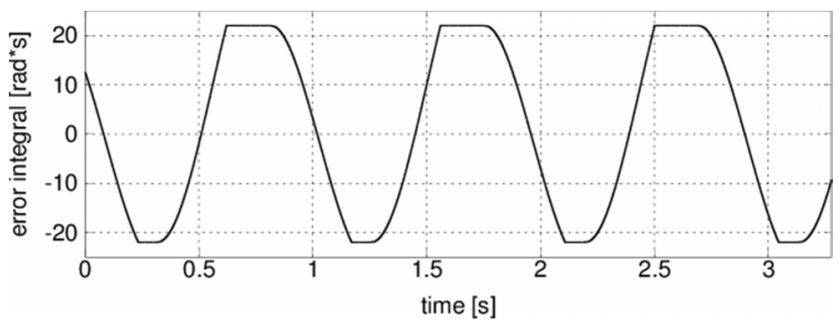

(c) Integral error.

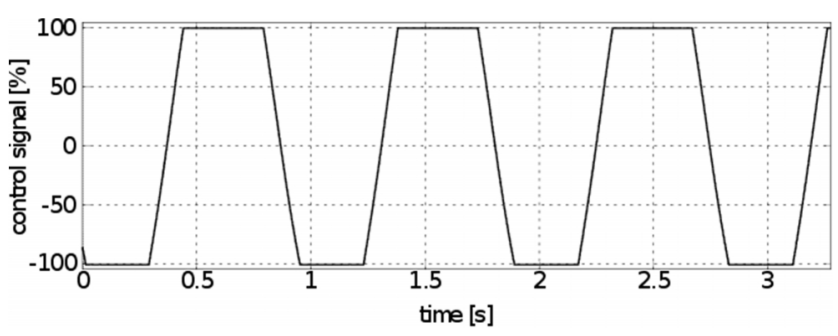

(d) Control signal.

Figure 7: System response when the integral error and control signal are saturated.
According to the simulation results obtained with the first order system, it is inferred that the oscillatory behavior of the motor can be understood by observing the phase portrait made with the integral error against the error signal as shown in Fig. 8.

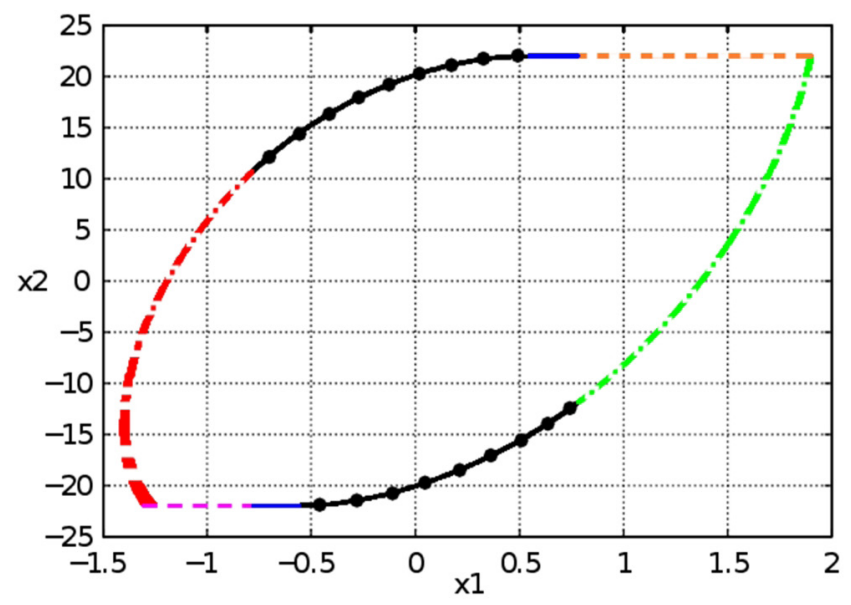

Figure 8: Contribution of each configuration in the formation of the periodic orbit in a PI servocontroler for a DC motor.

\section{CONCLUSIONS}

Saturations produce abrupt changes in the system configurations. These changes enrich the variety of system responses. In this paper, it is shown the effects of saturations in a PI controller for a first order system through simulations. The model based on Finite State Machines offers a systematic way to deal with this kind of dynamical systems that present abrupt changes in their configurations. Also, it has been presented experimental results of a PI servovontroller for a DC motor that let confirm the presence of the same configurations found for the first order system. In both cases, it is shown how the contribution of each configuration yields the system dynamics to an isolated periodic orbit.

The literature has reported that oscillations can occur when PI control system has an unstable equilibrium. In this work, we have shown that a PI control system can oscillate even though the system is stable in a neighborhood of an equilibrium point. 


\section{ACKNOWLEDGEMENTS}

We acknowledge the financial support by the DIMA (Dirección de Investigaciones de Manizales - Universidad Nacional de Colombia) through the HERMES project codes 12475 and 13750. Guillermo Gallo also thanks to Colciencias for the financial support to his master studies through the program Jovenes investigadores.

\section{BIBLIOGRAPHY}

1. M. Kothare, P. Campo, M. Morari and C. Nett, “A unified framework for the study of anti-windup designs, in Automatica, Elsevier Science B.V., 1994; 30, pp.1869-1883.

2. E. Freire, E. Ponce and J. Ros, "Limit cycle bifurcation in SISO control systems with saturation", in Proceedings of the 16th IFAC World Congress, Prague, 2005; 16, 1.

3. I. Moreno and R. Suárez, "Existence of periodic orbits of stable saturated systems", Systems \& Control Letters, Elsevier B.V., 2004; 51, pp.293-309.

4. J. Aracil, E. Ponce and T. Alamo, "A frecuency-domain approach to bifurcations in control systems with saturations", International Journal of Systems Science, Taylor \& Francis Ltd, 2000; 31(10), pp.1261-1271.

5. E. Ponce, J. Aracil and D. Pagano, "Control Systems with Actuator Saturation and Bifurcations at Infnity", in Proceedings of the 7th Mediterranean Conference on Control and Automation, Israel, 1999; pp.1598-1608.

6. Z. Lin, "Global Control of Linear Systems with Saturating Actuators", Proceedings of the 35th Conference on Decision and Control, Japan, dec 1996.

7. A. Saberi, Z. Lin and A. Teel, "Control of Linear Systems with Saturating Actuators", IEEE transactions on Automatic Control, 1996; 41, 3.

8. K. Aström and L. Rundqwist, "Integrator windup and how to avoid it", In Proceedings of the 1989 American Control Conference, 1989; pp.16931698.

9. D. Bernstein and A. Michel, "A chronological bibliography on saturating Actuators", in International Journal of Robust and Nonlinear Control, 1995; 5, pp.375-380.

10. C. Bohn and D. Atherton, "An Analysis Package Comparing PID AntiWindup Strategies", in IEEE Control Systems, 1995; 15, 2, pp.34-40.

11. A. Visioli, "Modified anti-windup scheme for PID controllers", IEE Proceedings on Control Theory and Applications, 2003; 150(1), pp.49-54.

12. A. Scottedward Hodel and C. Hall, "Variable-Structure PID Control to Prevent Integrator Windup", IEEE Transactions on Industrial Electronics, 2001; 48(2), pp.442-451.

13. J. Öhr, "Anti-windup and control of systems with multiple input saturations: Tools, solutions and case studies", Ph.D. dissertation, Uppsala University, 2003.

14. L. Castaño and G. Osorio, "Design of a FPGA based position PI servo controller for a DC motor with dry friction", in Proceedings of the VII Southern Conference on Programmable Logic, Argetina, 2011; pp.75-80.

15. Joao Lima, Ricardo Menotti, Joao M. P.Cardoso, Eduardo Marques, "A Methodology to Design FPGA-based PID Controllers", in Proceedings of the IEEE International Conference on Systems, Man and Cybernetics, 2006. SMC'06., 2006; 3, pp.2577-2583.

16. J. Alvarez, E. Curiel and F. Verduzco, "Complex dynamics in classical control systems", Systems \& Control Letters, Elsevier B.V., 1997; 31, pp.277-285.

17. Y. Peng, D. Vrancic and R. Hanus, "Anti-windup, bumpless and conditioned transfer techniques for PID controllers", IEEE Control Systems, 1996; 16, 4, pp.48-57.

18. I. Moreno and R. Suárez. "Existence of periodic orbits of stable saturated systems", in Systems \& Control Letter, 2004; 51, pp. $293-309$. 